\title{
Inventario de coleópteros saproxílicos presentes en los pinares de Mallorca (Islas Baleares)
}

\author{
Núñez. L. ${ }^{1}$, Closa S. ${ }^{1}$, González, E. ${ }^{*}$, Lencina, J.L., ${ }^{3,4}$ Gallego, D. ${ }^{3,4}$ \\ ${ }^{1}$ Servei de Sanitat Forestal D.G. Espais Naturals i Biodiversitat, Conselleria de Medi Ambient, Agricultura i Pesca \\ (Govern de les Illes Balears). C/ Gremi de Corredors, $n^{\circ}$ 10, 1º, Polígono de Son Rossinyol, \\ 07009 - Palma- Illes Balears Tel.: 9711766 66, \\ ${ }^{2}$ SILCO, S.L. $n^{\circ} 12$ B, esc. 3, $2^{\circ}$ D, 28440, Guadarrama, Madrid \\ ${ }^{3}$ Departamento de Zoología y Antropología Física, Universidad de Murcia, \\ Campus Universitario de Espinardo. 30100 Murcia. \\ ${ }^{4}$ Sanidad Agrícola ECONEX, S.L., C/ Mayor, $n^{\circ} 15 B$ - Edificio ECONEX \\ Apartado de Correos $N^{\circ}$ 167, 30149 Siscar-Santomera, Murcia. \\ *Autor para correspondencia: lalogonzalezrosa@gmail.com
}

\section{Resumen}

En Mallorca la especie de conífera dominante es Pinus halepensis Mill. Una parte de los pinares de esta especie se encuentran en la Sierra de Tramuntana, incluida desde 2011 en la Lista del Patrimonio Mundial de la UNESCO. Desde 2010 se han llevado a cabo diversos trampeos con atrayentes, repartidos por la geografía mallorquina, con especial interés en la Sierra de Tramuntana. Algunos de ellos tenían como objetivo la reducción de población de escolítidos en áreas de riesgo, como la afectada por el incendio que tuvo lugar en agosto de 2013 y que calcinó algo más de 2000 ha de monte de alto valor ecológico, en los municipios de Andratx, Estellenç y Calvià. El empleo de trampas con atrayente de diversas especies de xilófagos, ha permitido conocer la diversa entomofauna de insectos saproxílicos presentes en estos ecosistemas y sus variaciones poblacionales tras episodios catastróficos como es el caso de los incendios. Se han inventariado las comunidades de coleópteros, así como determinado su fenología anual, mediante el seguimiento de capturas en trampeos destinados al control y seguimiento de Tomicus destruens y Monochamus galloprovincialis, éste último vector del nematodo de la madera del pino Bursaphelenchus xylophilus.

Palabras clave: Pinus halepensis, xilófagos, entomofauna, fenología. 


\section{Introducción}

Las sociedades humanas obtienen múltiples funciones de los ecosistemas forestales, ya que ayudan a regular el clima, proveen bienes como la madera, y sostienen altos valores de biodiversidad. Los bosques contribuyen a la dinámica del clima global a través del ciclo del carbono, representando importante reserva del mismo. Todas las formas de la deforestación, humanas y naturales, directamente provocan un impacto en el clima por la eliminación de esos sumideros de carbono. Las temperaturas han estado estrechamente vinculadas a las variaciones de dióxido de carbono atmosférico en un cambio cíclico que se repite a una escala de milenios (Walther et al., 2002). La vegetación se ha visto obligada a adaptarse a estos cambios cíclicos especialmente los bosques situados en las proximidades del ecotono forestal (Carrión et al., 2001). Esto causa debilitamiento y decaimiento del arbolado que los hace especialmente sensibles al ataque de insectos como los escolítidos. Pueden asociarse entonces ciertos brotes epidémicos a una consecuencia necesaria de un cambio climático que genera un clima más cálido (Dunn et al., 2006).

Un brote epidémico de escolítidos puede ser favorecido por factores ambientales como son los cambios de temperatura y la humedad, pero también las dinámicas de las relaciones insecto-árbol y otras relaciones interespecíficas insecto-insecto como las de depredación, parasitoidismo y competencia. De este modo ciertas especies verán favorecido su crecimiento y vigor en condiciones de temperatura más alta y otras sufrirán estés y decaimiento, que a su vez generará cambios bioquímicos que emitirán semioquímicos como etanol y $\alpha$-pineno (Kelsey et al., 2014), que serán detectados por algunos escolítidos como señales positivas de vulnerabilidad. Pero también pueden aparecer desfases entre ciclos biológicos. De modo que, por ejemplo, un depredador puede aparecer demasiado pronto o demasiado tarde como para controlar eficazmente la población de su especie presa, de modo que la presa salga favorecida por la menor presión de depredación. De acuerdo con Carnicer et al., (2009) las comunidades ecológicas, como la comunidad de saproxílicos forestales, son entidades complejas y dinámicas que están integradas por las poblaciones que a su vez son temporalmente variables e interactúan de maneras muy diversas. Ejemplos de estas interacciones son la competencia, depredación, herbivoría, dispersión y el parasitismo, entre otros. Esto causa continuos cambios en la arquitectura de los flujos de energía y de materia en el espacio y tiempo. Se hace necesario conocer esta complejidad, comenzando por describir a sus actores, para lo que los inventarios de entomofauna son una herramienta fundamental.

Se presentan los resultados de los inventarios de entomofauna asociada a los trampeos de seguimiento de las especies de insectos de interés forestal Monochamus galloprovincialis (Olivier, 1795), Tomicus destruens (Wollaston 1865) y Orthotomicus erosus (Wollaston, 1857), en Mallorca desde mediados de 2013 a fin de 2014. Se trata de los primeros esfuerzos en conocer la biodiversidad entomológica de insectos de la comunidad saproxílica forestal que incluyen las especies diana. Se pretende con ello aportar los primeros conocimientos sobre el grado de afección de estos trampeos sobre la biodiversidad entomológica de los pinares de las Islas Ba- 
leares, como herramienta de gestión de daños forestales en el marco de la conservación de la biodiversidad.

\section{Material y métodos}

\subsection{Seguimiento intensivo de Monochamus galloprovincialis (SIMG)}

Se instalaron dos trampas Crosstrap ${ }^{\circledR}$ (Econex, Murcia), en los términos municipales de Palma de Mallorca y Bunyola (Fig. 1), cebadas con atrayente cairo-feromonal Galloprotect 2D (SEDQ, Barcelona). Este cebo cuenta con la feromona de agregación 2-undeciloxil-1-etanol y las cairomonas ipsenol, 2-metil-3-buten-2-ol y $\alpha$-pineno. Las trampas fueron revisadas cada 10 días entre el 24 de octubre de 2013 y el 1 de mayo de 2014. Los cebos se repusieron cada 45 días de acuerdo con las indicaciones del fabricante.

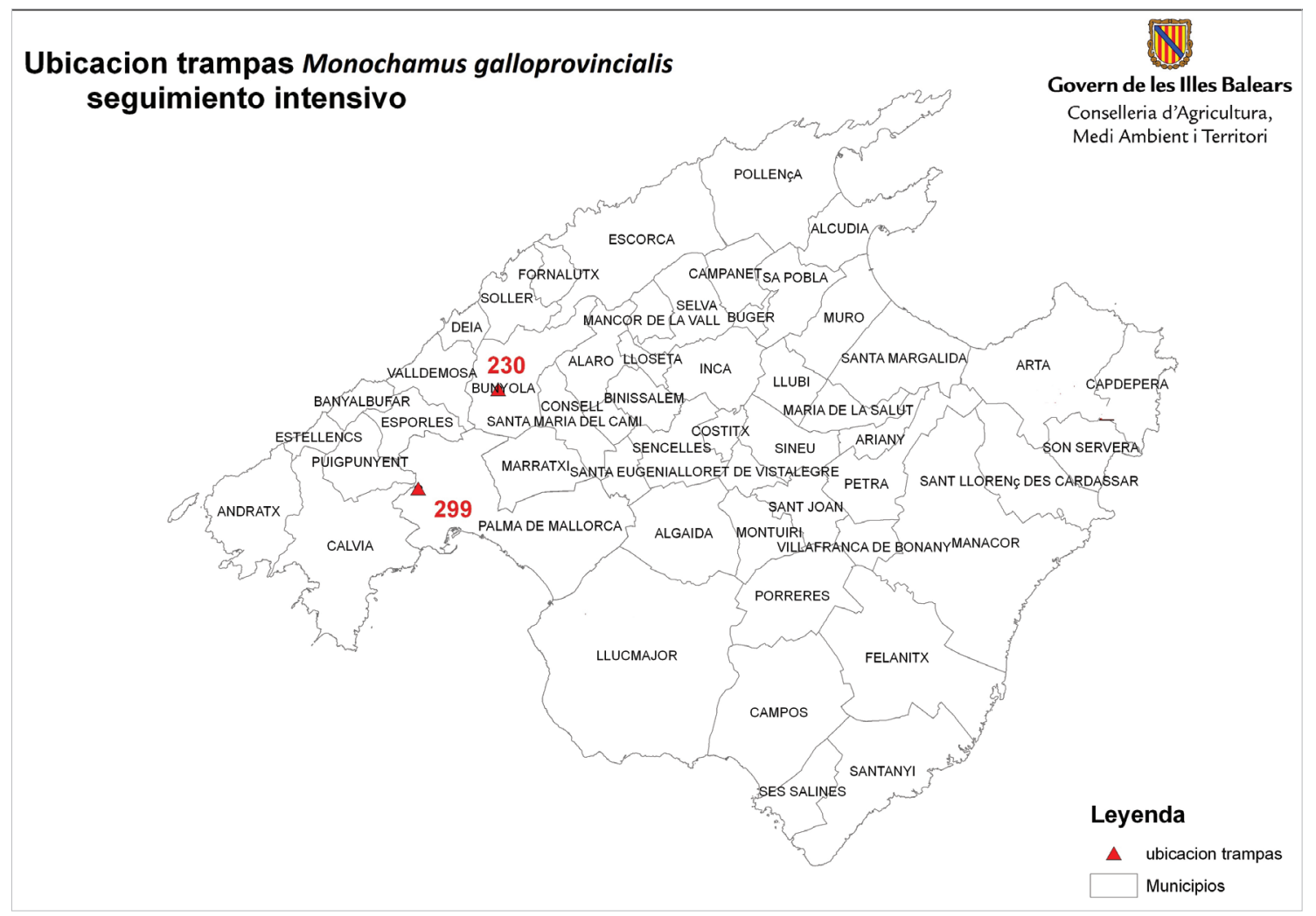

Figura 1. Ubicación de las trampas para seguimiento intensivo de Monochamus galloprovincialis (SIMG).

2.2. Seguimiento intensivo de los escolitidos Tomicus destruens y Orthotomicus erosus (SIMG)

Se instalaron cuatro trampas Theysohn, en los términos municipales de Palma 
de Mallorca, Bunyola, Llucmajor y Arta (Fig. 2). Las trampas fueron cebadas entre octubre de 2013 y mayo de 2014 con el atrayente comercial basado en las cairomonas etanol y $\alpha$-pineno ECONEX TOMICUS DESTRUENS (Econex, Murcia). Este atrayente se repuso cada dos meses, de acuerdo con las instrucciones de fabricante. El 1 de septiembre volvió a cambiarse el atrayente para T. destruens, manteniéndose hasta finales del mismo mes. Las trampas se revisaron cada 10 días.

Entre el 1 mayo y el 1 de septiembre de 2014, el atrayente fue sustituido por la feromona de agregación de $O$. erosus ECONEX ORTHOTOMICUS EROSUS (Econex, Murcia), compuesto por ipsenol, cis-verbenol y 2-metil-3-buten-2-ol, siendo sustituida cada 60 días, de acuerdo con las indicaciones del fabricante. Las trampas se revisaron igualmente cada 10 días.

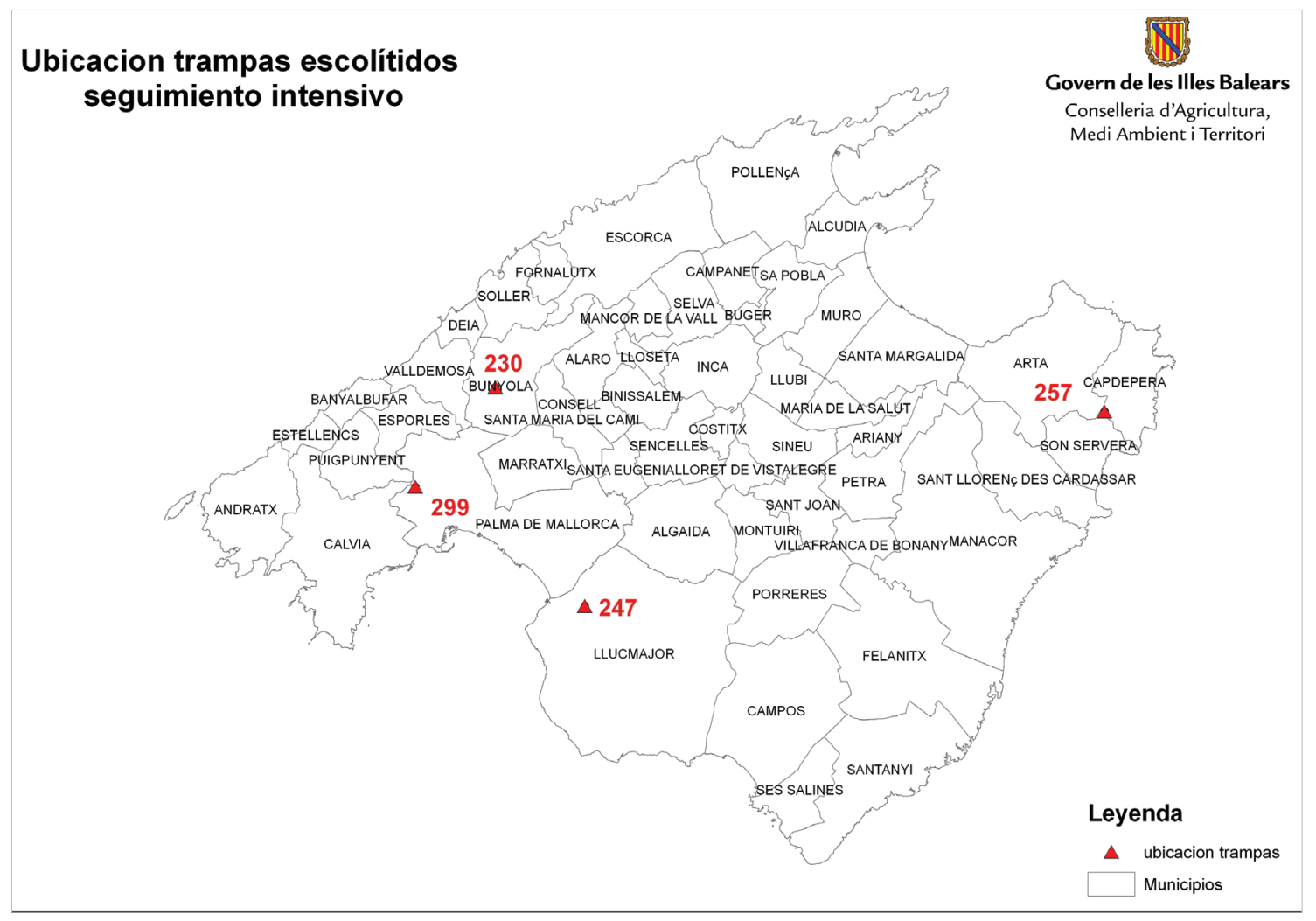

Figura 2. Ubicación de las trampas para seguimiento intensivo de los escolítidos Tomicus destruens y Orthotomicus erosus (SIES).

En ambos tipos de seguimiento se recurrió a la misma metodología para la determinación de las especies de la comunidad de saproxílicas: las muestras de campo eran conservadas en viales en etanol al $70 \%$ y transportados a laboratorio. Las muestras se limpiaron bajo agua corriente, y se realizó el recuento de todos los individuos, identificando su especie, bajo lupa binocular con zoom de hasta 80 aumentos. En caso necesario se recurrió a la extracción y examen de la genitalia masculina o femenina para completar la determinación. 


\section{Resultados}

El recuento total de ejemplares en los seguimientos fue de 8038 individuos, 5485 en el SIMG y 2553 ejemplares en el SIES. Los ejemplares se han distribuido en 19 familias de Coleoptera (Tab. 1), a razón de 17 en el SIMG y 19 en el SIES. El número total especies ha sido de 53, a razón de 43 especies en el trampeo de SIMG y de 53 especies en el SIES.

La familia más representada es Curculionidae, con 10 especies, todas pertenecientes a la subfamilia Scolytinae, habiéndose capturado 8 especies en SIMG y 10 especies en SIES. Dos familias han registrado 6 especies, Cerambycidae, con 6 especies capturadas en ambos inventarios y Tenebrionidae, con 5 especies en SIMG y 6 especies en SIES. La familia Carabidae está representada por 5 especies en ambos inventarios, mientras de Bostrichidae e Histeridae, ambas con 4 especies en total, están representadas con 3 y 2 especies en SIMG, respectivamente, mientras que en SIES se han encontrado el total de especies en ambos casos. El resto de familias están representadas por una o dos especies. Únicamente Laemophloeidae y Oedemeridae no están representadas en SIMG.

Tabla 1. Repartición de especies por familias, en función de los inventarios.

\begin{tabular}{lccc}
\hline \multicolumn{1}{c}{ Familia } & Número de especies & & \\
\hline \multicolumn{1}{c}{ SIMG } & SIES & Total \\
\hline Bostrichidae & 3 & 4 & 4 \\
Buprestidae & 2 & 2 & 2 \\
Carabidae & 5 & 5 & 5 \\
Cerambycidae & 6 & 6 & 6 \\
Cleridae & 2 & 2 & 2 \\
Coccinellidae & 1 & 1 & 1 \\
Curculionidae & 8 & 10 & 10 \\
Dasytidae & 1 & 1 & 1 \\
Elateridae & 1 & 1 & 1 \\
Endomychidae & 1 & 1 & 1 \\
Eucinetidae & 1 & 1 & 1 \\
Histeridae & 2 & 4 & 4 \\
Laemophloeidae & 0 & 1 & 1 \\
Lampyridae & 1 & 1 & 1 \\
Lyctidae & 1 & 1 & 1 \\
Monotomidae & 1 & 1 & 1 \\
Mycetophagidae & 1 & 1 & 1 \\
Oedemeridae & 0 & 1 & 1 \\
Ptinidae & 1 & 2 & 6 \\
Tenebrionidae & 5 & 6 & 1 \\
Zopheridae & 1 & 1 & 6 \\
\hline
\end{tabular}


Tabla 2. Lista de especies registrada por inventario. Las especies sombreadas en gris constituyen citas relevantes para las Islas Baleares (ver más detalles en el texto).

\begin{tabular}{|c|c|c|c|}
\hline Familia & ESPECIE & SIMG & SIES \\
\hline \multirow[t]{4}{*}{ Bostrichidae } & Scobicia chevrieri (Villa \& Villa 1835) & $\mathrm{X}$ & $\mathrm{X}$ \\
\hline & Sinoxylon sexdentatum (Olivier 1790) & & $\mathrm{X}$ \\
\hline & Xylopertha praeusta (Germar, 1817) & $\mathrm{X}$ & $\mathrm{X}$ \\
\hline & Xyloperthella picea (Olivier, 1790) & $\mathrm{X}$ & $\mathrm{X}$ \\
\hline \multirow[t]{2}{*}{ Buprestidae } & Acmaeodera (Palaeotethya) bipunctata (Olivier, 1790) & $\mathrm{X}$ & $\mathrm{X}$ \\
\hline & Anthaxia (Melanthaxia) rugicollis (Lucas 1849) & $\mathrm{X}$ & $\mathrm{X}$ \\
\hline \multirow[t]{5}{*}{ Carabidae } & Dromius (Dromius) agilis (Fabricius 1787) & $\mathrm{X}$ & $\mathrm{X}$ \\
\hline & Dromius (Dromius) meridionalis Dejean 1825 & $\mathrm{X}$ & $\mathrm{X}$ \\
\hline & Microlestes abeillei (Brisout de Barneville 1885) & $\mathrm{X}$ & $\mathrm{X}$ \\
\hline & Microlestes reitteri (Holdhaus 1912) & $\mathrm{X}$ & $\mathrm{X}$ \\
\hline & Pseudoophonus (Pseudoophonus) rufipes (De Geer, 1774) & & $\mathrm{X}$ \\
\hline \multirow{6}{*}{ Cerambycidae } & Acanthocinus griseus (Fabricius 1792) & $\mathrm{X}$ & $\mathrm{X}$ \\
\hline & Arhopalus ferus (Mulsant, 1839) & $\mathrm{X}$ & $\mathrm{X}$ \\
\hline & Arhopalus syriacus (Reitter 1895) & $\mathrm{X}$ & $\mathrm{X}$ \\
\hline & Monochamus galloprovincialis (Olivier 1795) & $\mathrm{X}$ & $\mathrm{X}$ \\
\hline & Oxypleurus nodieri (Mulsant 1839) & $\mathrm{X}$ & $\mathrm{X}$ \\
\hline & Pogonocherus perroudi (Mulsant 1839) & $\mathrm{X}$ & $\mathrm{X}$ \\
\hline \multirow[t]{2}{*}{ Cleridae } & Opilo domesticus (Sturm 1837) & $\mathrm{X}$ & $\mathrm{X}$ \\
\hline & Thanasimus formicarius (Linnaeus 1758) & $\mathrm{X}$ & $\mathrm{X}$ \\
\hline Coccinellidae & Rhyzobius chrysomeloides (Herbst 1792) & $\mathrm{X}$ & $\mathrm{X}$ \\
\hline \multirow[t]{10}{*}{ Curculionidae } & Chaetoptelius vestitus (Mulsant \& Rey, 1860) & & $\mathrm{X}$ \\
\hline & Crypturgus mediterraneus (Eichhoff 1871) & $\mathrm{X}$ & $\mathrm{X}$ \\
\hline & Hylastes linearis (Erichson 1836) & $\mathrm{X}$ & $\mathrm{X}$ \\
\hline & Hylurgus ligniperda (Fabricius 1787) & $\mathrm{X}$ & $\mathrm{X}$ \\
\hline & Hylurgus miklitzi (Wachtl 1881) & $\mathrm{X}$ & $\mathrm{X}$ \\
\hline & Orthotomicus erosus (Wollaston 1857) & $\mathrm{X}$ & $\mathrm{X}$ \\
\hline & Pteleobius kraatzi (Eichhoff, 1864) & & $\mathrm{X}$ \\
\hline & Tomicus destruens (Wollaston 1865) & $\mathrm{X}$ & $\mathrm{X}$ \\
\hline & Xyleborinus saxesenii (Ratzeburg 1837) & $\mathrm{X}$ & $\mathrm{X}$ \\
\hline & Xyleborus eurygraphus (Ratzeburg 1837) & $\mathrm{X}$ & $\mathrm{X}$ \\
\hline Dasytidae & Dasytes (Dasytes) terminalis Jacquelin Du Val 1861 & $\mathrm{X}$ & $\mathrm{X}$ \\
\hline Elateridae & Lacon punctatus (Herbst 1779) & $\mathrm{X}$ & $\mathrm{X}$ \\
\hline Endomychidae & Symbiotes gibberosus (Lucas 1846) & $\mathrm{X}$ & $\mathrm{X}$ \\
\hline Eucinetidae & Nycteus meridionalis (Laporte de Castelnau 1836) & $\mathrm{X}$ & $\mathrm{X}$ \\
\hline \multirow{4}{*}{ Histeridae } & Cylister elongatus (Thunberg 1787) & & $\mathrm{X}$ \\
\hline & Cylister filiformis (Erichson 1834) & & $\mathrm{X}$ \\
\hline & Plegaderus (Plegaderus) otti Marseul 1856 & $\mathrm{X}$ & $\mathrm{X}$ \\
\hline & Teretrius (Neotepetrius) parasita (Marseul, 1862) & $\mathrm{X}$ & $\mathrm{X}$ \\
\hline Laemophloeidae & Cryptolestes ferrugineus (Stephens 1831) & & $\mathrm{X}$ \\
\hline Lampyridae & Nyctophila heydeni (Olivier, 1884) & $\mathrm{X}$ & $\mathrm{X}$ \\
\hline Lyctidae & Trogoxylon impressum (Comolli 1837) & $\mathrm{X}$ & $\mathrm{X}$ \\
\hline Monotomidae & Rhizophagus (Eurhizophagus) depressus (Fabricius 1792) & $\mathrm{X}$ & $\mathrm{X}$ \\
\hline Mycetophagidae & Litargus (Litargus) connexus (Geoffroy 1785) & $\mathrm{X}$ & $\mathrm{X}$ \\
\hline Oedemeridae & Nacerdes (Xanthochroa) raymondi canyellesi Vázquez 2001 & & $\mathrm{X}$ \\
\hline \multirow{2}{*}{ Ptinidae } & Ernobius lucidus (Mulsant \& Rey, 1863) & & $\mathrm{X}$ \\
\hline & Stagetus franzi Español 1969 & $\mathrm{X}$ & $\mathrm{X}$ \\
\hline \multirow[t]{6}{*}{ Tenebrionidae } & Corticeus linearis (Fabricius, 1790) & & $\mathrm{X}$ \\
\hline & Corticeus pini (Panzer 1799) & $\mathrm{X}$ & $\mathrm{X}$ \\
\hline & Hymenorus doublieri (Mulsant, 1851) & $\mathrm{X}$ & $\mathrm{X}$ \\
\hline & Isomira (Isomira) melanophthalma (Lucas 1846) & $\mathrm{X}$ & $\mathrm{X}$ \\
\hline & Lagria grenieri (Brisout, 1868) & $\mathrm{X}$ & $\mathrm{X}$ \\
\hline & Nesotes viridicollis (Schaufuss 1869) & $\mathrm{X}$ & $\mathrm{X}$ \\
\hline Zopheridae & Aulonium ruficorne (Olivier, 1790) & $\mathrm{X}$ & $\mathrm{X}$ \\
\hline
\end{tabular}


La lista completa de especies puede consultarse en la Tab. 2. El Eucetínido Nycteus meridionalis (Laporte de Castelnau 1836), el ptínido Stagetus franzi Español 1969 y el tenebriónido Corticeus linearis (Fabricius, 1790) constituyen nuevas citas para las Islas Baleares.

La cita de la subespecie de Oedemérido Nacerdes (Xanthochroa) raymondi canyellesi Vázquez 2001 es relevante por representar la segunda cita de este insecto tras su descripción como subespecie en 2001.

\section{Discusión}

La comunidad de sasproxílicos, constituida por 53 especies no es excesivamente compleja si se compara con los resultados obtenidos en otros pinares del levante peninsular, como las 272 especies encontradas en pinares de la región de Murcia (Gallego and Campo 2010). Esto representa aproximadamente el $20 \%$ de las especies. De cualquier modo estos datos son poco comparables ya que los seguimientos de Murcia se mantuvieron durante 4 años y comprendían una variabilidad de pinares que no existe en Islas Baleares. Por otro lado también es esperable que el efecto de insularidad redunde en un menor número de especies.

El número de 19 familias inventariadas representa aproximadamente el 30\% de las familias inventariadas en Murcia (61 familias), aunque las consideraciones de tiempo de muestreo, diversidad de ambientes e insularidad antes comentadas para el nivel de especie también resultan válidas para el nivel de familia.

Respecto a la rareza de estas especies y familias, en su mayoría se trata de especies de amplia distribución en los pinares ibéricos. De cualquier modo es destacable la cita de la subespecie de Oedemérído Nacerdes raymondi canyellesi, una especie saproxílica con larva considerada como depredador facultativo de escolítidos. Esta subespecie fue descrita en Palma de Mallorca en 2001 (Vázquez, 2001) y no había sido capturado desde entonces.

\section{Conclusiones}

Se han aportado los primeros datos de comunidades de saproxílicos de pinares baleares. Aunque la comunidad es relativamente sencilla en comparación con resultados de inventarios sistemáticos y de larga duración en otros pinares del levante ibérico, muy posiblemente esto se deba al bajo número de trampas utilizado, el escaso tiempo de trampeo y un muy posible efecto de insularidad.

\section{Bibliografía}

Carnicer, J., Jordano, P., Melián, C.J. 2009. The temporal dynamics of resource use by frugivorous birds: a network approach. Ecology, 90:7-15 
Carrión, J.S., Munuera, M., Dupré, M., Andrade, A. 2001. Abrupt vegetation changes in the Segura mountains of southern Spain throughout the Holocene. J. Ecol., 89: 783-797

Dunn, D., Crutchfield, J.P. 2006. Insects, Trees, and Climate: The bioacoustic ecology of deforestation and entomogenic climate change. Santa Fe Institute Working Paper, 120: 2332

Gallego D, Campo M.T. (2010) El bosque ante el cambio climático. Murcia en clave ambiental 25, 18-29.

Kelsey, R.G., Gallego, D., Sánchez-García, F.J., Pajares J.A. 2014. Ethanol accumulation during severe drought may signal tree vulnerability to detection and attack by bark beetles. Can. J. For. Res. 44: 554-561

Vázquez, X.A. 2001. Una nueva especie de Nacertes (Xanthochroa) raymondi (Mulsant \& Godart, 1860) de la isla de Mallorca (Coleoptera: Oedemeridae). Boll. Soc. Hist. Nat. Balears, 49: 39-44.

Walther G.R., Post, E., Convey, P., Menzel, A., Parmesan, C., Beebee, T., Fromentin, J.M., Hoegh-Guldberg, O., Wairlein, F. 2002 Ecological responses to recent climate change. Nature, 416: 389-395 